\title{
PORTING AND TESTING NPOESS CRIMSS EDR ALGORITHMS
}

\author{
Susan Kizer $^{1}, X u$ Liu $^{2}$ \\ 1 Science Systems Applications Intl., Hampton, VA 23666 USA \\ 2 NASA Langley Research Center, Hampton, VA 23681 USA
}

\begin{abstract}
1. ABSTRACT
As a part of the National Polar-orbiting Operational Environmental Satellite System (NPOESS) and the NPOESS Preparatory Project (NPP), the instruments Cross-track Infrared Sounder (CrIS) and Advanced Technology Microwave Sounder (ATMS) make up the Cross-track Infrared and Microwave Sounder Suite (CrIMSS). CrIMSS will primarily provide global temperature, moisture, and pressure profiles and calibrated radiances [1]. In preparation for the NPOESS/NPP launch, porting and testing of the CrIMSS Environmental Data Record (EDR) algorithms need to be performed.
\end{abstract}

CrIMSS EDR algorithms in operational code (Raytheon) and in science code (Northrop Grumman Application System (NGAS)) have been made available by the Integrated Program Office (IPO) [2]. The science code is written in Fortran and requires a static database of input parameters and dynamic input in the form of Science Data Records (SDRs) and auxiliary information such as surface pressure and scene type. The operational code executes the EDR algorithms in Fortran, representative of the science code, but uses a C++ wrapper to perform pre-processing and post-processing functions. See Figure 1 for a diagram of the CRIMSS EDR operational code layout. The Version 1.5 operational code was made functional in a Linux/Unix environment by performing the following alterations:

a. removed $\mathrm{C}++$ portion not related to CrIMSS

b. removed $\mathrm{C}++$ references to dynamic memory storage (DMS) and replaced with static, SDR and auxiliary input data readers, and removed information messaging (INF), both not fully functional as provided in v1.5

c. added ability to run Fortran-only version of operational code (comparable to science code), can easily convert between operational code and Fortran-only version for code testing

d. added functionality to read data in either Fortran or $\mathrm{C}++$ (science or operational) format therefore adding the ability to test both science and ops code with the same input data. 
In order to test the CrIMSS EDR algorithms performance, dynamic input in the form of SDR and auxiliary data need to be made available. Atmospheric InfraRed Sounder (AIRS) Level 2 data provide realistic variations of the input parameters needed for simulation of ATMS/CrIS spectra: atmospheric temperature, moisture, and ozone profiles, cloud top and cloud fractions for each FOV, microwave liquid cloud water amounts, and surface emissivities for both MW and IR [4]. Auxiliary data such as surface pressure and scene type are also provided by AIRS Level 2 data files. AIRS Level 2 data was input to the CrIMSS radiative transfer models to generate a selfconsistent simulated CrIS and ATMS SDR dataset. CrIMSS EDR operational code is then used to perform retrievals with good convergence. Figures 2 and 3 show the AIRS and CrIMSS temperature and moisture error for a one granule comparison. The SDR simulated data generated will aid in operational code validation and controlled identification of algorithm issues, but the simulated test scenes have limitations in regards to cloud simulation, surface emissivity data, trace gases profiles and spectroscopic errors.

The Infrared Atmospheric Sounding Interferometer (IASI) on board the METOP-A satellite provides all the information needed to generate CrIS proxy data. The IASI instrument is a Fourier Transform Spectrometer (FTS) with a spectral sample interval of $0.25 \mathrm{~cm}^{-1}$ and a continuous spectral coverage from 645 to $2760 \mathrm{~cm}^{-1}$. The CrIS instrument has three spectral bands with spectral coverage of $648.75-1096.25 \mathrm{~cm}^{-1}, 1207.5-1752.5 \mathrm{~cm}^{-1}$, and 2150.0 - $2555.0 \mathrm{~cm}^{-1}$, respectively [5]. The transformation from an IASI radiance spectrum to a CrIS proxy spectrum is a rigorous mathematical operation. The IASI instrument has 3 spectral bands and each spectral band has 4 pixels within the AMSU field of views (FOV). The CrIS instrument has 3 spectral bands and each spectral band has 9 FOV within a $45 \mathrm{~km}$ field of regard (FOR). A spatial linear interpolation is performed to convert the 4 IASI pixels to the 9 CrIS pixels. The ATMS proxy data are generated according to algorithms described in reference xxxx Proxy data generated from IASI/AMSU/MHS data provide highly realistic SDR proxy data for CrIMSS. Both IASI and CrIS are Fourier Transform spectrometers. CrIS spectra can be accurately generated through a rigorous mathematical transformation as described by Liu et al (2009). See Figure 4 for IASI and CrIS proxy spectra. The generated proxy data have been successfully ingested into the CrIMSS OPS code. This data set will be very useful for algorithm testing and validation because it provides realistic scenes (atmosphere, surface and cloud) which are based on observations. It will help us identify areas of deficiencies in both forward and inverse models, testing robustness of the operational code, check error handling capability of the OPS code. It will be used for bias characterization and algorithm tuning, and EDR performance evaluations.

\section{CONCLUSIONS}

The CrIMSS operational code has been successfully ported into various LINUX systems. They are modified in such a way that the results can be compared with that of the science code. We have generated proxy data simulated by using the CrIMSS forward models and the AIRS Level 2 data products. The CrIMSS EDR performance can be evaluated using this proxy data set. We have also created another proxy data set which is 
based on real IASI/AMSU/MHS observations. This data set provides us realistic spectra which is very close to that which will be observed by the CRIMSS instrument. Lessons will be learned from this data set about forward model errors, algorithm tuning, emissivity constraints, etc.

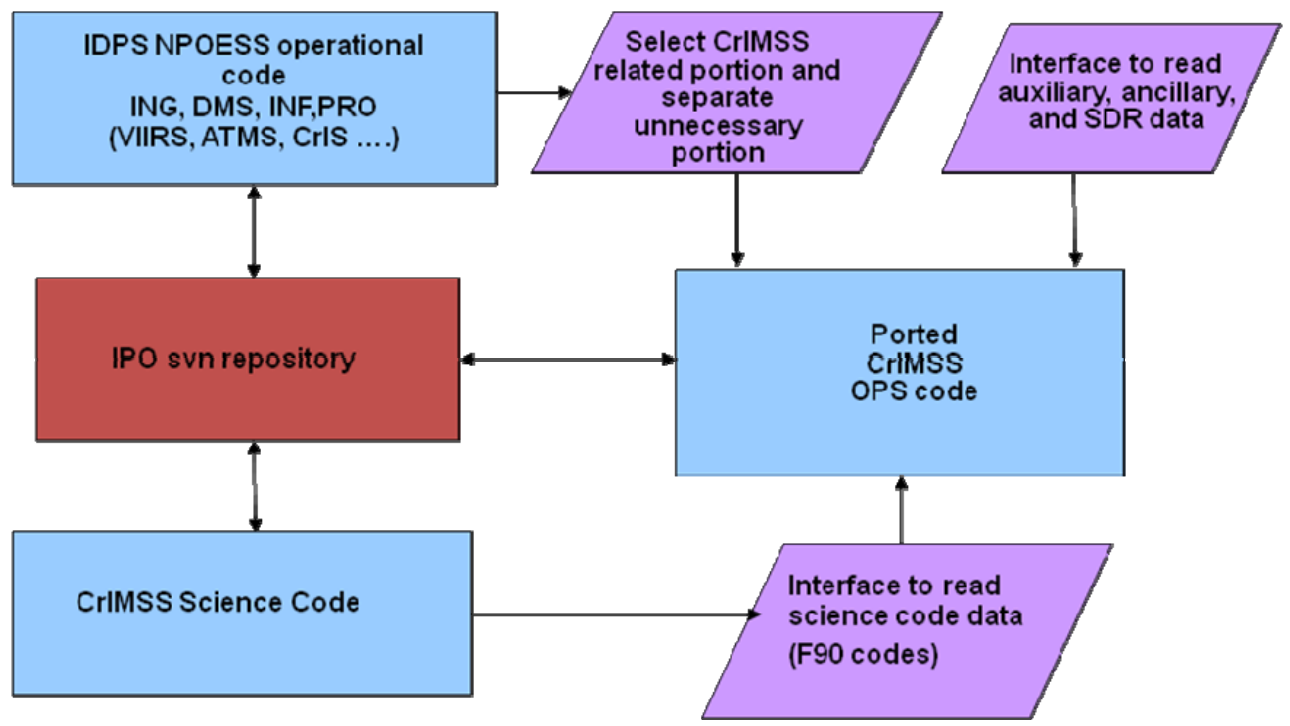

Figure 1: Layout of CrIMSS EDR Operational Code
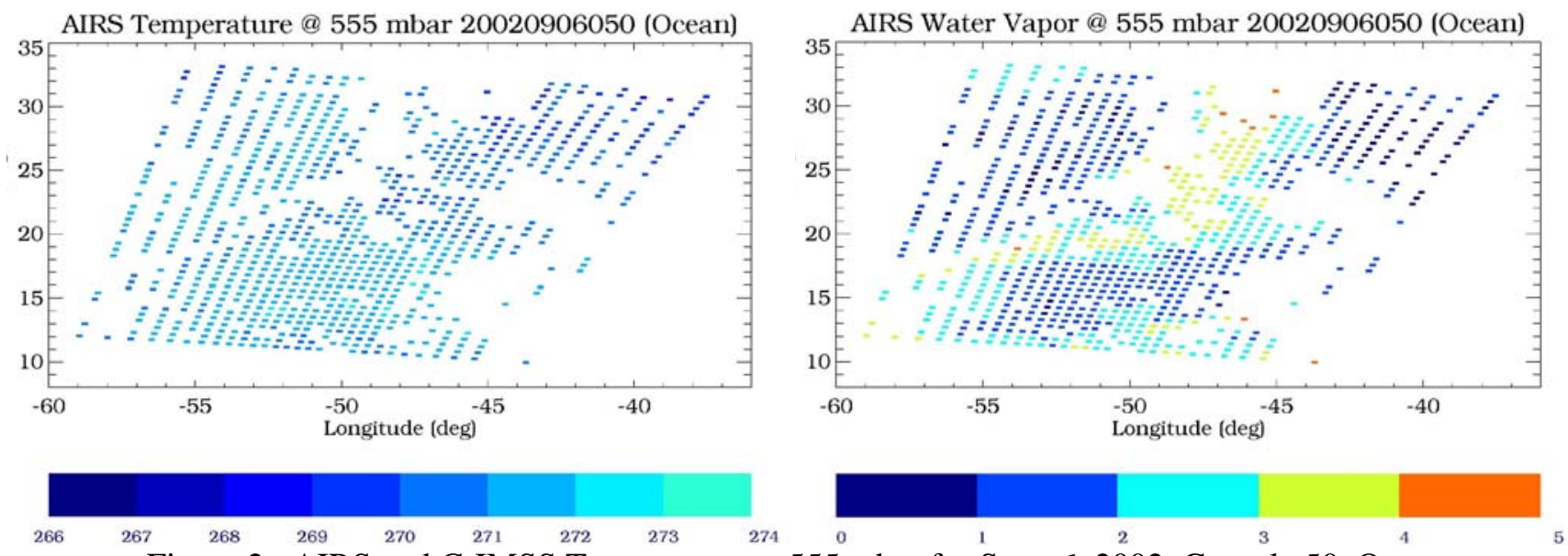

Figure 2: AIRS and CrIMSS Temperature at 555 mbar for Sept. 6, 2002, Granule 50, Ocean 

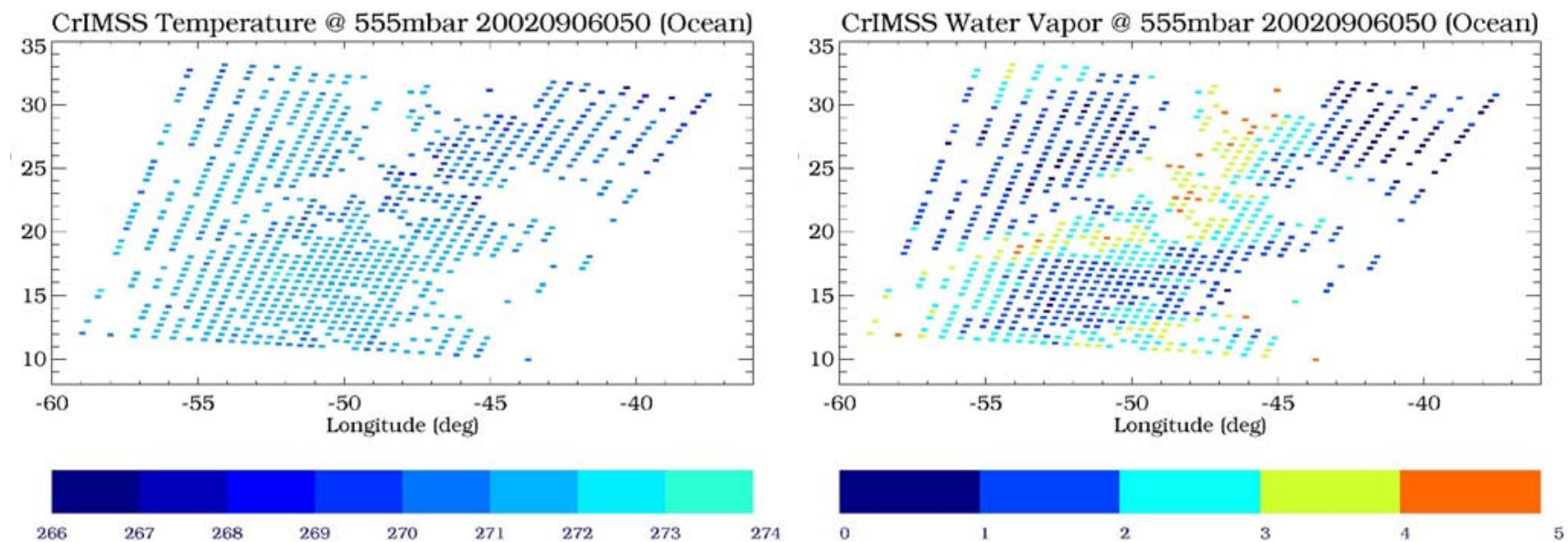

Figure 3: AIRS and CrIMSS Moisture at 555 mbar for Sept. 6, 2002, Granule 50, Ocean

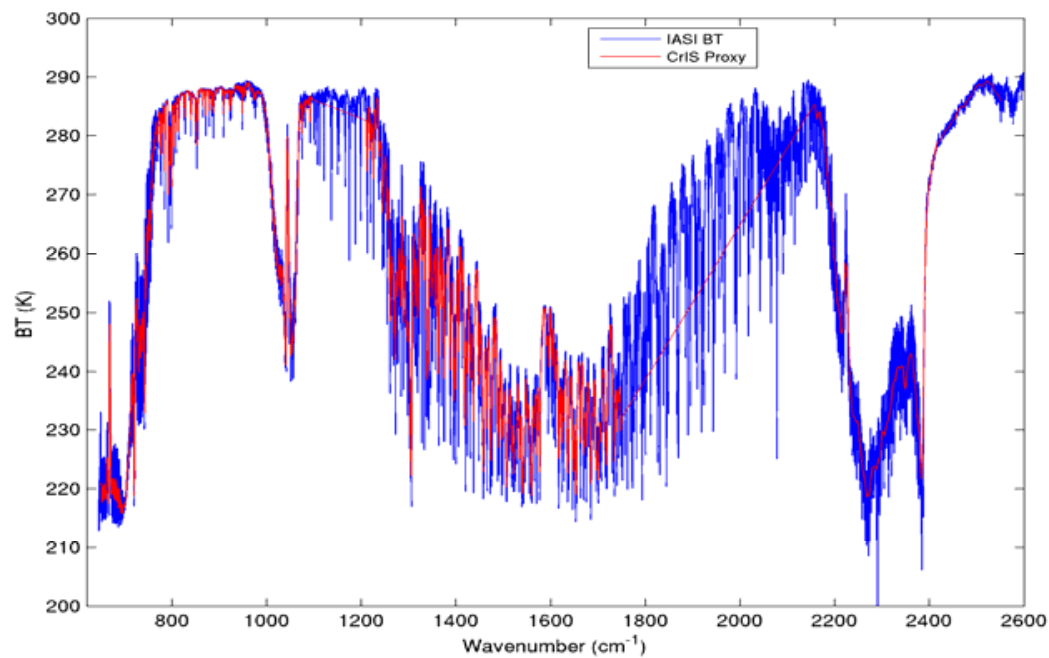

Figure 4: An example of high resolution IASI spectrum and generated CrIS proxy spectrum using double FFT method

\section{REFERENCES}

[1] http://ipo.noaa.gov

[2] Northrop Grumman Space Technology, “Cross Track Infrared Sounder (CrIS) Volume II, Environmental Data Records (EDR) Algorithm Theoretical Basis Document ATBD”, Northrop Grumman Space Technology, Redondo Beach, CA, Feb. 8, 2007

[3] X. Liu, and S. Kizer, “Testing CrIMSS EDR Algorithm Using Synthetic and Proxy Data”, Sounder Operational Algorithm Team (SOAT) Meeting, Logan, UT, September 10, 2009. ftp://www.star.nesdis.noaa.gov/pub/smcd/spb/nnalli/SOAT/2009-09/090910soat_04_04_liu.pdf

[4] M. T. Chahine , M. Gunson, et.al., “AIRS Level 2 Algorithm Theoretical Basis Document Version 2.2”, JPL D-17006, Version 2.2, April 26, 2001

[5] EUMETSAT, "IASI Level 1 Product Guide”, EUM/OPS-EPS/MAN/04/0032, Issue v3, May 13, 2009

[6] B. Blackwell, “An Update On ATMS Testing And Cal/Val Planning”, Sounder Operational Algorithm Team (SOAT) Meeting, Logan, UT, September 10, 2009

Acknowledgements: Thanks to the financial and logistics support from NASA and the IPO and interactions with NGAS and AER. 